\title{
Weight Loss in Microemulsion with Eugenol Oil
}

\author{
C.R LAILI*, S HAMDAN and N KAMARUDDIN \\ School of Fundamental Science, Universiti Malaysia Terengganu, \\ 21030, K.Terengganu, Terengganu, Malaysia. \\ *Correspondence author E-mail: Iaili@umt.edu.my \\ http://dx.doi.org/10.13005/ojc/320543
}

(Received: July 17, 2016; Accepted: September 26, 2016)

\begin{abstract}
Essential oil has been widely used in many products. In this work, the location of the microemulsion region with eugenol was studied in two different surfactants namely sodium dodecyl sulphate, SDS (ionic) and Tween 80 (non-ionic). The phase diagrams were constructed for water/ SDS:hexanol (30:70)/eugenol oil and water/ Tween 80 / eugenol oil systems by titrating to turbidity. The result showed that microemulsion regions were present in both systems with the SDS:hexanol system formed a larger water-in-oil microemulsion region compared to Tween 80 system. In order to study the behaviour of the microemulsion with eugenol oil subjected to several conditions such as evaporation and rheology test, several weight ratios of eugenol oil to surfactants were studied. The weight loss during evaporation was carried under ambient condition. The rheological behaviour was also observed in both systems. The evaporation rate for the microemulsion with SDS was found to be higher than the equivalent counterpart but with Tween 80 . The rheology study showed that the flow is of Newtonian behaviour with little or no shear thinning
\end{abstract}

Keywords: Microemulsion; Phase diagrams; Non-ionic surfactant.

\section{INTRODUCTION}

It is well known many plants produce important organic compounds such as oils, gum, waxes, pharmaceutical as well as medicinal'. With the advances in equipment and cutting edge technologies, new methods have been developed to extract this biologically active plant-derived chemicals as witnessed today ${ }^{2}$. One of the important compound is the essential oil. Essential oils are the volatile components and they are liquid, volatile, transparent, and soluble in lipid and organic solvents. It is also less density than water. All part of the plants can be used to extract the essential oils including, buds, stems, flowers, leaves, twigs, seeds, fruits, roots, wood and secretory cells ${ }^{2}$. They have been used in many fields since then for example, as antimicrobial, analgesic, sedative, anti-inflammatory, spasmolytic, and anesthetic $c^{1,3,4}$ and with latest application in alcohol free microemulsion ${ }^{5}$. It is also have been used to protect the plants from herbivores but also attract insect as their agent to spread their pollens and seeds ${ }^{2}$. 
The use of essential oil or perfumery in microemulsion systems have received much attention $^{6-9}$. Microemulsions have always been a striking area in colloid chemistry due to their importance in vast area of industrial application. Microemulsions were brought to the attention of the scientific community beginning in the late 1940's by Schulman and a series of collaborators ${ }^{10-12}$. Since then numerous attempts have been made to investigate various aspects of microemulsions from the treatment of microemulsions as colloidal systems ${ }^{13,14}$ to more theoretical contributions and their dynamics ${ }^{15-17}$. Microemulsions have merits over other vehicles or solvents due to their improved stability, solubilisation characteristics and ease in preparation. Depending on the region of the microemulsions, eugenol in microemulsions have many industrial applications such as cosmetic, coatings, preservatives, flavours, disinfectants and aromatherapy ${ }^{4,18-21}$.

This work it is not meant to be argumentative to the work previously done on microemulsion and essential oils, but to report the region of microemulsion with eugenol using two different surfactants, its water loss during evaporation and its rheology behavior. We believe the findings will contribute further the understanding of the interaction of essential oil, and especially eugenol in microemulsion systems of relevance to the area of fragrance.

\section{MATERIAL AND METHOD}

The surfactants sodium dodecyl sulphate (SDS) (>99\% and polyoxyetehylene (20) sorbitan monooleate (Tween 80) (95\%) (HLB 15.0); were purchased from Sigma-Aldrich. 1-hexanol (>98\%) and pure eugenol $(99 \%)$ were also purchased from Sigma-Aldrich. All components were used as received without further purification. The water used throughout the study was double distilled.

\section{Construction of phase diagrams}

The phase diagrams were determined on a clear/turbid criteria basis by mixing two of the components and titrating with the smallest amount of the third component. The samples were then thoroughly mixed to homogeneity with a vortex mixer, centrifuged and then allowed to reach equilibrium at a specific temperature in a water bath. The phases were then examined by visual inspection between cross polarisers. An estimated region of the phases can then be made by this method by noting the turbid and clear compositions.

\section{Evaporation of Microemulsion}

Samples of microemulsion from different weight ratios of oil to surfactants were prepared. The samples were poured on a watchglass and were kept in a humidity chamber (Eureka Model RT-48C) to control the convection movement from the surroundings. The experiment was monitored at ambient humidity and at room temperature. The weight loss was weighed at $5 \mathrm{~min}$. interval time for 10 hours. Repeated determinations at different days gave identical results.

\section{Rheological Measurements}

A strain-controlled rheometer model HAAKE RheoStress 600 was used for oscillatory shear experiments in cone-plane geometry. Temperature was set to $\mathrm{T}=25^{\circ} \mathrm{C}$ using cone plate spindle 51 . The flow curve of the microemulsions was generated with a shear rate of $250 \mathrm{rpm}$ in 120 seconds. The measurements were made by shearing at maximum shear and returning to zero within seconds in order to obtain the ascendant and descendent curves, respectively.

\section{RESULT AND DISCUSSION}

\section{Phase Diagram}

The aim of the construction of ternary

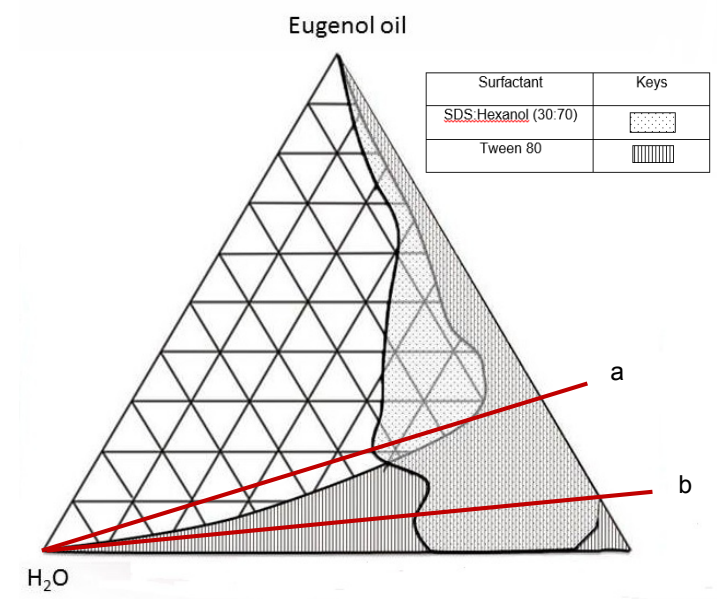

Fig. 1: Comparison of phase diagram between SDS and Tween 80 system 
phase diagrams was to locate the region of microemulsions for the aqueous mixture of eugenol oil with surfactants at different weight ratios. After considering various factors the pseudoternary phase diagrams consisting of water, surfactants and essential oil were constructed.

\section{Aqueous surfactant-eugenol system}

The two surfactants used throughout this investigation are anionic surfactant SDS and the nonionic surfactant Tween 80 . The ratio for SDS to hexanol was chosen to be $30: 70$ by weight. The weight ratio of 30:70 was chosen since it exhibited the highest water solubility in the system consisting of water/hexanol/SDS. The solubility regions for the aqueous isotropic regions are given in Fig. 1 for the SDS and Tween 80 systems. Both of the systems show an isotropic region that is extended continuously from the pure eugenol to the surfactant in a crescent manner towards the water apex.

However, eugenol is not completely miscible in the SDS system, that is, it is only up to about 85 percent by weight. The maximum solubility of water is also observed to be higher in the Tween 80 system. This is illustrated by the continuous

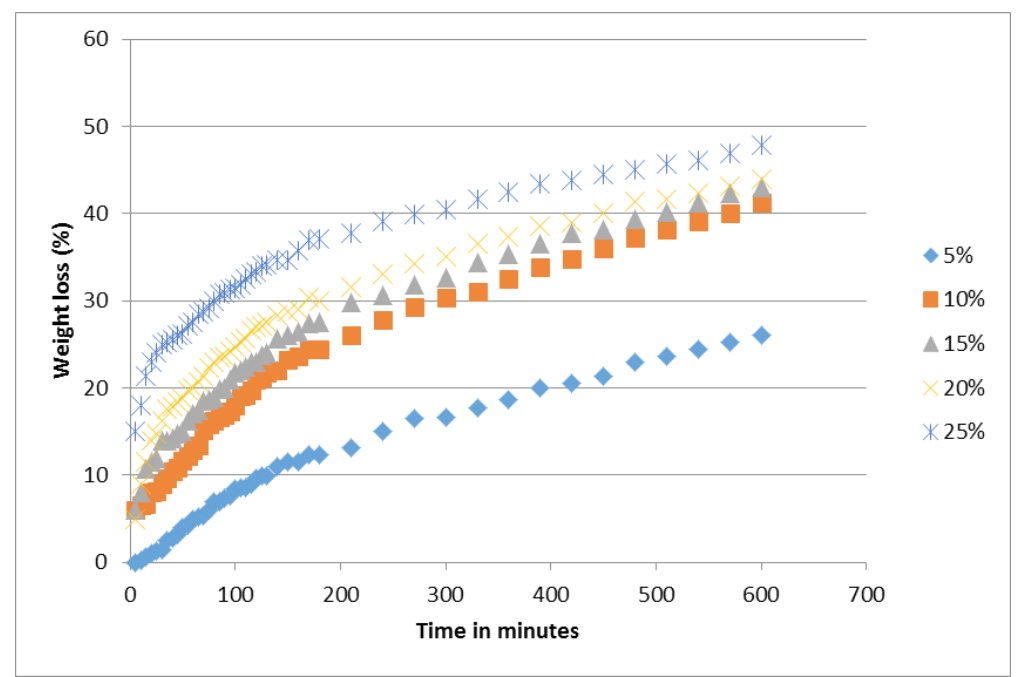

Fig. 2: Evaporation rate of aqueous system of eugenol/SDS:1-hexanol at weight ratio of 30/70

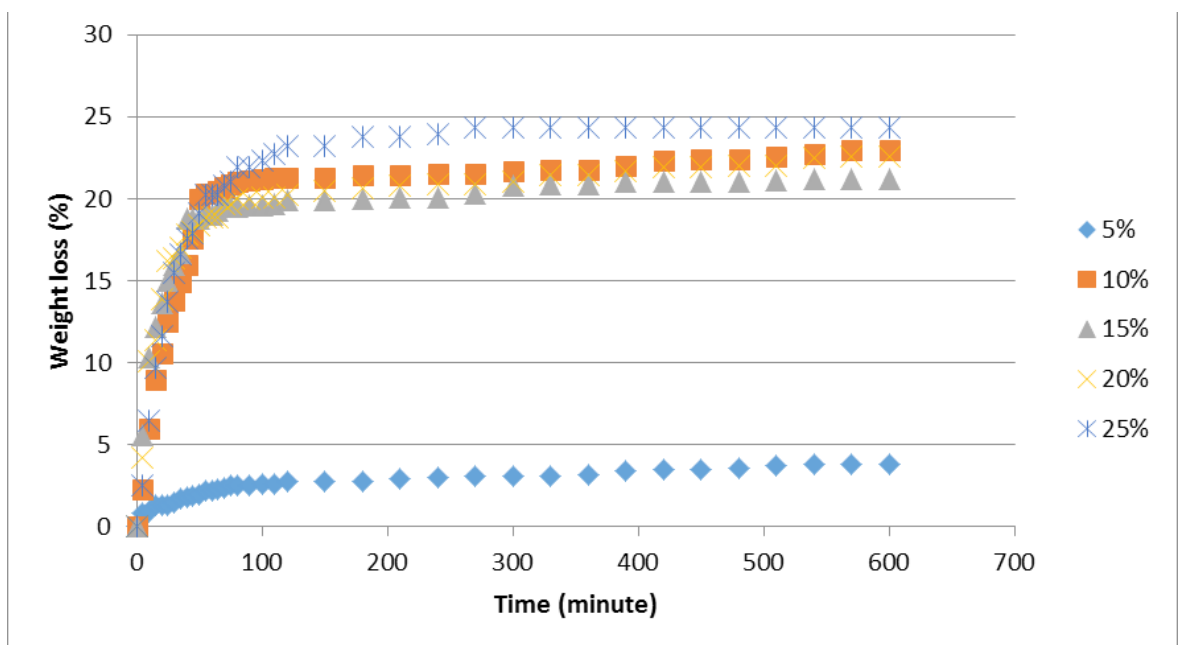

Fig. 3: Evaporation rate of aqueous system of eugenol/Tween 80 at weight ratio of 10/90 
solubility region in convex manner from the eugenol apex until the water apex (Fig. 1). The maximum solubility of water in the SDS system is limited to about 33 percent at a mixture of eugenol and SDS at weight ratio of 30:70 (solid line of Fig. 1). These phenomenon are interesting and can be explained by the hydrophilicity of the surfactants. Tween 80 having a HLB value 18 should exhibit higher solubiliy in water in the presence of oil compared to SDS. Since SDS is mixed with hexanol, the polar group of hexanol decrease the hydrophilicity of SDS and it leads to the decreasing of the ability of the SDS molecules to solubilize with water.

\section{Weight Loss during Evaporation}

The evaporation was performed for components across the microemulsion region which exhibit maximum water solubility for each surfactant systems as shown on lines (a) and (b) for SDS and Tween 80 (Fig. 1). The water content for each system varies between 5 to 25 percent by weight.

For the SDS system, the compositions by weight of oil/surfactant is 30:70 (line (a) of Fig. 1). A plot of the weight loss for this system is shown in Fig. 2. It is observed that the weight loss in this composition increased linearly in the interval used

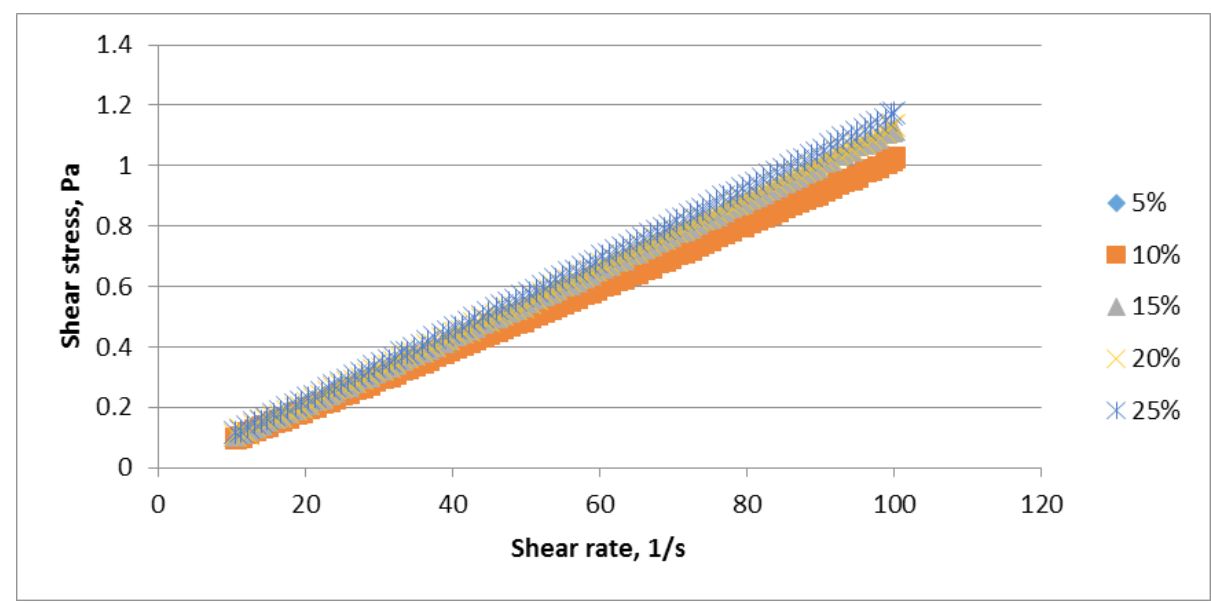

(a)

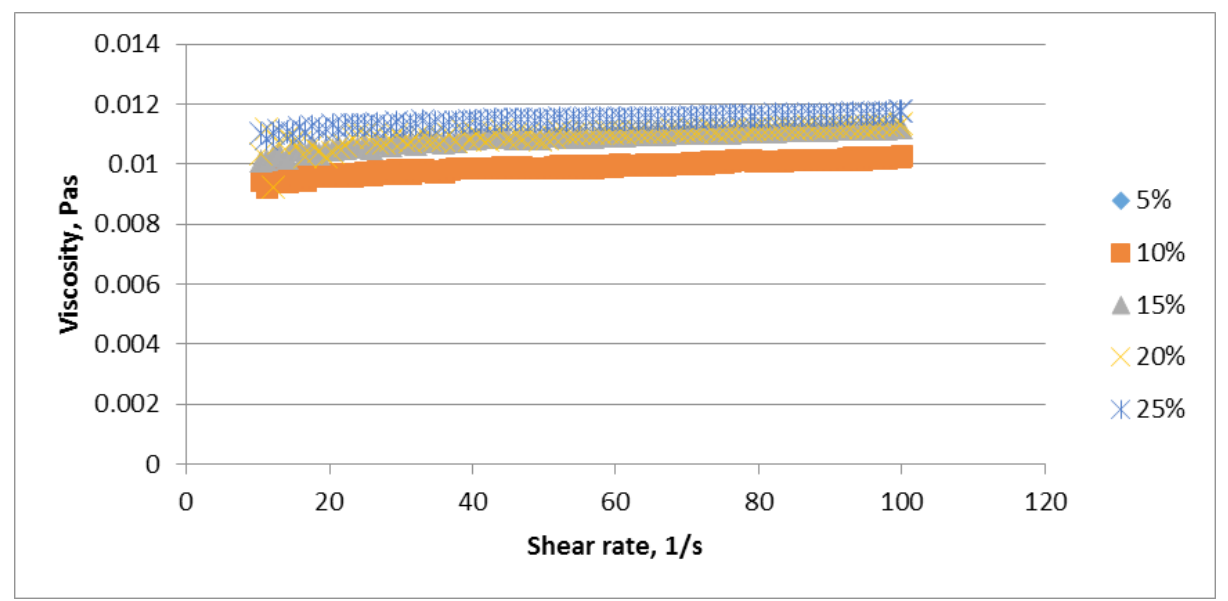

(b)

Fig. 4: Flow curves, shear stress (a) and viscosity (b) versus shear rate for SDS system 


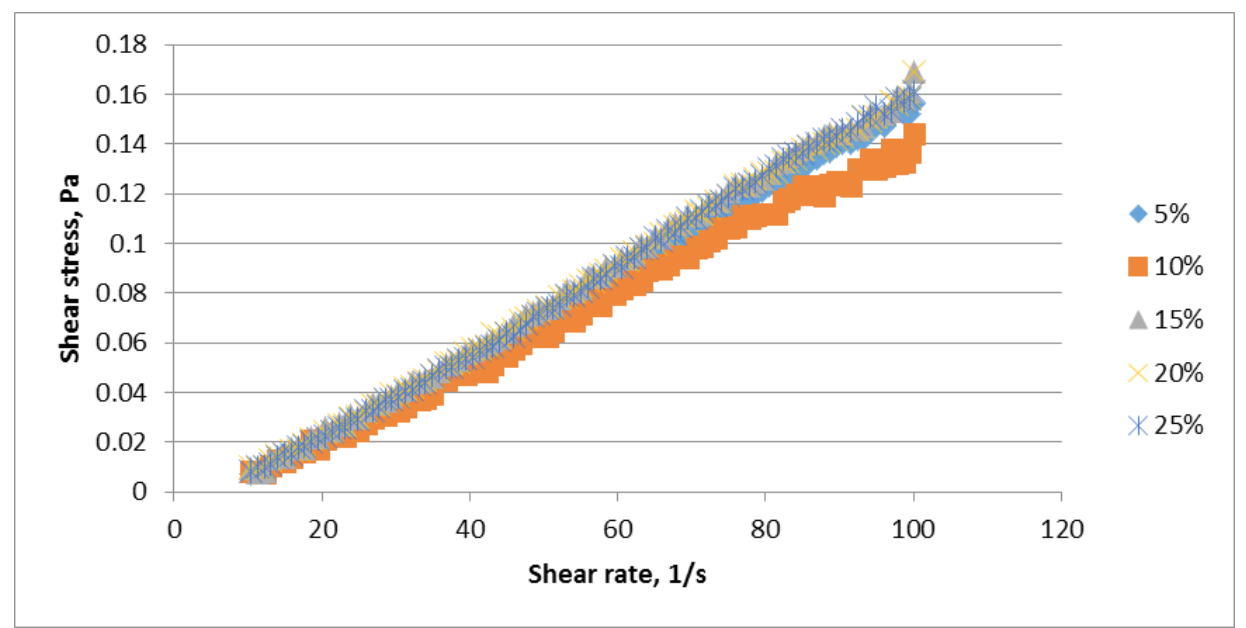

Fig. 5: Flow curves, shear stress versus shear rate for Tween 80 system

with main characteristics. A higher slope at the early stage of evaporation followed by a sudden turn in the slope at about 200 min reaching almost a plateau. This implies that the evaporation rate occurs at two stages, that is, the initial rate is faster than the later one. The curves also show that the evaporation rate increase with the increment of water content. This can be explained by the possible presence of surfactant aggregates at higher water content

Fig. 3 show equivalent data but for aqueous systems with Tween 80 . It shows similar trend as previously exhibited by the SDS counterpart. It shows an initial increase in the weight loss with time but the sudden turn occurs much early after less than $100 \mathrm{~min}$, resulting in a more plateaued progression. The sudden increase in the weight loss after 5 percent by weight of water is very interesting. This could be explained by the presence of surfactant aggregate such as inverse micelles which may take many shapes at higher water content. Comparison with equivalent systems with SDS shows a much lower weight loss for the Tween 80 system.

\section{Rheological Measurement}

The rheological behaviour was observed on the same weight ratios as that for the evaporation test. Fig. $4 \mathrm{a}$ and $\mathrm{b}$ show the plotted shear stress versus shear rate and viscosity versus shear rate for SDS. The rheology profile obtain is expected which is Newtonian behavior as the shear stress observe show linear correlation and the viscosity were constant against the shear rate ${ }^{22}$. The constant value observe indicates maybe weak or no shear thinning. Same phenomenon is observe for the equivalent data but with a much lower sheer stress and viscosity at the interval investigated for the Tween 80 system (Fig. 5).

\section{CONCLUSION}

In summary, the location of the microemulsion region with eugenol and its evaporation rate together with its rheology behaviour in two surfactant systems have been determined. The result demonstrated that the type of surfactants to be crucial in the formation and size of microemulsion region with eugenol. The SDS system showed a larger microemulsion region but with a limited maximum water solubility when compared to Tween system. It also showed a higher water loss during evaporation, suggesting that the aggregates formed in the microemulsion by the aqueous SDS molecules has lower hydrophilicity and lower vapour pressure ${ }^{23}$. The rheology measurements confirmed that both of the systems exhibit Newtonian behaviour as predicted and that little or no shear shinning effect exhibited.

\section{ACKNOWLEDGEMENT}

This work is supported by Fundamental Research Grant from Ministry of Higher Education, Malaysia (Grant No. 59365) and is gratefully acknowledged. 


\section{REFERENCES}

1. Balandrin, M.F.; Klocke, J.A.; Wurtele, E.S.; Bollinger, W. H. Science, 1985., 228(4704), 1154-1160,

2. Bakkali, F.; Averbeck, S.; Averbeck, D., Food and Chemical Toxicology. 2008., 46, 446475,

3. Graven, E.H.; Deans, S.G.; Svoboda, K.P.; Mavi, S.; Gundidza, M.G., Flavour and Fragrance Journal, 1992., 7(3), 121-123,

4. Lee, M.; Choi, J.; Posadzki, P.; Ernst, E. Maturitas, 2012., 71, 257-260,

5. Hamed, S.F.; Sadek, Z.; Edris, A. Journal of Oleo Science, 2012., 61(11), 641-648,

6. Tokuoka, Y.; Uchiyama, H.; Abe, M.; Ogino, K. Journal of Colloid and Interface Science. 1990., 152 (2), 402-409, 1990,

7. Hamdan, S.; Ahmad, F.B.H.; Dai, Y.Y.; Dzulkefly, K.; Ku Bulat, K.H. Effect of Journal of Dispersion Science and Technology, 1999., 20(1-2), 415-423,

8. Friberg, S.E.; Huang, T.; Fei, L.; Vona, Jr.; S.A.; Aikens, P.A., Progress in Colloid and Polymer Science, 1996., 101, 18-22,

9. Zhang, L.; Critzer, F.; Davidson, P.M.; Zhong, Q. Food Chemistry, 2014., 165: 113-118,

10. Hoar, T.P \& Schulman, J.H. Nature (London), 1943., 152, 102-103,

11. Schulman, J.H.; Riley, D.P. Journal of Colloid and Interface Science, 1948.,3,83-405,

12. Schulman, J.H.; Friend, J.A. Journal of Colloid and Interface Science, 1949., 4, 497-509,

13. Adamson, A.W., Colloid Interface Sci., 29, 261-267, 1969,

14. Ahmad, S.I.; Shinoda, K.; Friberg, S.E. J Colloid Interface Sci., 1974.,47, 32-37,

15. Reiss, H., Journal of Colloid and Interface Science, 1975., 53, 61-70,

16. Sjoblom, J.; Blom, C.; Stenius, P. Acta Chemica Scandinavica A, 1986., 40, 306312 ,

17. Sjoblom, J.; Lindberg, R.; Friberg, S.E. Advances in Colloid and Interface Science, 1996., 95, 125-287,

18. Rodriguez, A.; Batlle, R.; Nerin, C., Progress in Organic Coating, 2007.,60(1), 33-38,

19. Edris, A.E.; El-Galeel, M.A.S. World Applied Sciences Journal, 2010., 8(1), 86-91,

20. Warnke, P.; Becker, S.; Podschun, R.; Sivanathan, S.; Springer, R.; Russo, P.; Wiltfang, J.; Fickenser, H.; Sherry, E. Journal of Cranio-Maxillofacial Surgery, 2010., 37, 392-397,

21. Kamatou, G. P.; Alvaro, M. J.; Vermaak, I. Molecules, 2012., 17,6953-6981,

22. Gregory, G.W.; Chen, C.M. The Journal of Physical Chemistry. 1992.,96(23), 94929497,

23. Li, P.; Han, B.; Yan, H.; Liu, R., Journal of Chemical Engineering Data, 1996.,41, 285286 , 